\title{
Exact Solutions of Coupled Sine-Gordon Equations Using the Simplest Equation Method
}

\author{
Yun-Mei Zhao \\ Department of Mathematics, Honghe University, Mengzi, Yunnan 661100, China \\ Correspondence should be addressed to Yun-Mei Zhao; zhaoyunmei2000@126.com
}

Received 27 November 2013; Accepted 9 January 2014; Published 16 February 2014

Academic Editor: Ray K. L. Su

Copyright (C) 2014 Yun-Mei Zhao. This is an open access article distributed under the Creative Commons Attribution License, which permits unrestricted use, distribution, and reproduction in any medium, provided the original work is properly cited.

The simplest equation method has been used for finding the exact solutions of coupled sine-Gordon equations. Such equations have some useful applications in physics and biology, so finding their exact solutions is of great importance.

\section{Introduction}

Recently, the coupled sine-Gordon equations

$$
\begin{array}{r}
u_{t t}-u_{x x}=-\delta^{2} \sin (u-\omega) \\
\omega_{t t}-\alpha^{2} \omega_{x x}=\sin (u-\omega) \\
\alpha>0, \delta>0,
\end{array}
$$

have been introduced by Khusnutdinova and Pelinovsky [1]. The coupled sine-Gordon equations generalize the FrenkelKontorova dislocation model $[2,3]$. System (1) with $\alpha=1$ was also proposed to describe the open states in DNA model [4].

Very recently, system (1) was studied by many researchers and various methods. It was studied by Salas, using a special rational exponential ansatz [5]. Zhao et al. obtained some new solutions including Jacobi elliptic function solutions, hyperbolic function solutions, and trigonometric function solutions by the Jacobi elliptic function expansion method [6], the hyperbolic auxiliary function method [7], and the symbolic computation method [8].

In the past four decades, the study of nonlinear partial differential equations (NLEEs) modelling physical phenomena has become an important research topic. Seeking exact solutions of NLEEs has long been one of the central themes of perpetual interest in mathematics and physics. With the development of symbolic computation packages like Maple and Mathematica, many powerful methods for finding exact solutions have been proposed, such as the homogeneous balance method $[9,10]$, the auxiliary equation method $[11,12]$, the Exp-function method [13, 14], the Darboux transformation $[15,16]$, the tanh-function method [17], and the $\left(G^{\prime} / G\right)$ expansion method $[18,19]$.

The simplest equation method is a very powerful mathematical technique for finding exact solutions of nonlinear ordinary differential equations. It has been developed by Kudryashov [20,21] and used successfully by many authors for finding exact solutions of ODEs in mathematical physics $[22,23]$.

In this paper, we will apply the simplest equation method [24] to obtain some new and more general explicit exact solutions of the coupled sine-Gordon equations.

\section{The Simplest Equation Method}

In this section, we will give the detailed description of the simplest equation method.

Step 1. Suppose that we have a nonlinear partial differential equation (PDE) for $u(x, t)$ in the form

$$
N\left(u, u_{t}, u_{x}, u_{t t}, u_{x t}, u_{x x}, \ldots\right)=0 \text {, }
$$

where $N$ is a polynomial in its arguments. 
Step 2. By taking $u(x, t)=u(\xi), \xi=x-c t$, we look for traveling wave solutions of (2) and transform it to the ordinary differential equation (ODE)

$$
N\left(u,-c u^{\prime}, u^{\prime}, c^{2} u^{\prime \prime},-c u^{\prime \prime}, u^{\prime \prime}, \ldots\right)=0 .
$$

Step 3. Suppose that the solution $u$ of (3) can be expressed as a finite series in the form

$$
u=\sum_{i=0}^{n} A_{i}(H(\xi))^{i}
$$

where $H(\xi)$ satisfies the Bernoulli or Riccati equation, $n$ is a positive integer that can be determined by balancing procedure [21], and $A_{i}(i=0,1,2, \ldots, n)$ are parameters to be determined.

The Bernoulli equation we consider in this paper is

$$
H^{\prime}(\xi)=a H(\xi)+b H^{2}(\xi),
$$

where $a$ and $b$ are constants. Its solutions can be written as

$$
\begin{aligned}
H(\xi) & =\frac{-a D_{1}}{b\left(D_{1}+\cosh (a(\xi+C))-\sinh (a(\xi+C))\right)}, \\
H(\xi) & =\frac{-a(\cosh (a(\xi+C))+\sinh (a(\xi+C)))}{b\left(D_{2}+\cosh (a(\xi+C))+\sinh (a(\xi+C))\right)},
\end{aligned}
$$

where $D_{1}, D_{2}$, and $C$ are constants.

For the Riccati equation

$$
H^{\prime}(\xi)=a H^{2}(\xi)+b H(\xi)+s,
$$

where $a, b$, and $s$ are constants, we will use the solutions

$$
\begin{gathered}
H(\xi)=-\frac{b}{2 a}-\frac{\theta}{2 a} \tanh \left[\frac{\theta}{2}(\xi+C)\right], \\
H(\xi)=-\frac{b}{2 a}-\frac{\theta}{2 a} \tanh \left(\frac{\theta}{2} \xi\right) \\
+\frac{\operatorname{sech}(\theta \xi / 2)}{C \cosh (\theta \xi / 2)-(2 a / \theta) \sinh (\theta \xi / 2)},
\end{gathered}
$$

where $\theta^{2}=b^{2}-4 a s$.

Step 4. Substituting (4) into (3) with (5) (or (7)), then the left hand side of (3) is converted into a polynomial in $H(\xi)$, and equating each coefficient of the polynomial to zero yields a set of algebraic equations for $A_{i}, a, b(i=0,1,2, \ldots, n)$. Solving the algebraic equations by symbolic computation, we can determine those parameters explicitly.

Step 5. Assuming that the constants $A_{i}, a, b(i=0,1,2, \ldots, n)$ can be obtained in Step 4 and substituting the results into (4), then we obtain the exact traveling wave solutions for (2).

Remark 1. In (5), when $a=A$ and $b=-1$ we obtain the Bernoulli equation

$$
H^{\prime}(\xi)=A H(\xi)-H^{2}(\xi) .
$$

Equation (9) admits the following exact solutions:

$$
H(\xi)=\frac{A}{2}\left(1+\tanh \left(\frac{A}{2}(\xi+C)\right)\right),
$$

when $A>0$, and

$$
H(\xi)=\frac{A}{2}\left(1-\tanh \left(\frac{A}{2}(\xi+C)\right)\right),
$$

when $A<0$.

\section{Exact Solutions of the Coupled Sine-Gordon Equations}

In this section, we solve the coupled sine-Gordon equations by the simplest equation method.

In order to solve (1), we introduce a new unknown function $\varphi=\varphi(x, t)$ by the formula

$$
\varphi(x, t)=u(x, t)-\omega(x, t),
$$

so that $\omega(x, t)=u(x, t)-\varphi(x, t)$. According to (1), we have

$$
\begin{gathered}
u_{t t}-u_{x x}=-\delta^{2} \sin \varphi, \\
u_{t t}-\varphi_{t t}-\alpha^{2}\left(u_{x x}-\varphi_{x x}\right)=\sin \varphi .
\end{gathered}
$$

Let

$$
\begin{gathered}
\xi=\mu(x-c t), \\
\varphi=2 \arctan (v(\xi))=2 \arctan (v(\mu(x-c t))),
\end{gathered}
$$

then

$$
\sin \varphi=\sin (2 \arctan v(\xi))=\frac{2 v(\xi)}{1+v^{2}(\xi)} .
$$

Substitution (14)-(15) into (13), we get the following coupled system of nonlinear differential equations:

$$
\begin{aligned}
& \mu^{2}\left(c^{2}-1\right)\left(1+v^{2}(\xi)\right) u^{\prime \prime}(\xi)+2 \delta^{2} v(\xi)=0, \\
& 2 \mu^{2}\left(c^{2}-\alpha^{2}\right) v^{2}(\xi)\left(u^{\prime \prime}(\xi)-v^{\prime \prime}(\xi)\right) \\
& \quad+\mu^{2}\left(c^{2}-\alpha^{2}\right)\left(u^{\prime \prime}(\xi)-2 v^{\prime \prime}(\xi)\right) \\
& +\mu^{2}\left(c^{2}-\alpha^{2}\right) v^{4}(\xi) u^{\prime \prime}(\xi) \\
& +v(\xi)\left(4 \mu^{2}\left(c^{2}-\alpha^{2}\right) v^{\prime 2}(\xi)-2\right)-2 v^{3}(\xi)=0 .
\end{aligned}
$$

According to the first equation of (16), we have

$$
u^{\prime \prime}(\xi)=-\frac{2 \delta^{2} v(\xi)}{\mu^{2}\left(c^{2}-1\right)\left(1+v^{2}(\xi)\right)} .
$$

Substituting (17) into the second equation of (16), we obtain a single nonlinear second-order differential equation in the unknown $v=v(\xi)$ :

$$
\begin{aligned}
& \left(c^{2}-1\right) \mu^{2}\left(c^{2}-\alpha^{2}\right)\left(v^{2}(\xi)+1\right) v^{\prime \prime}(\xi) \\
& \quad-2 \mu^{2}\left(c^{2}-1\right)\left(c^{2}-\alpha^{2}\right) v(\xi) v^{\prime 2}(\xi) \\
& \quad+\left(v^{3}(\xi)+v(\xi)\right)\left(\delta^{2}\left(c^{2}-\alpha^{2}\right)+c^{2}-1\right)=0 .
\end{aligned}
$$


As we can see, it suffices to find analytic solutions to (18). Observe that if $v(\xi)$ is a solution of (18), then $-v(\xi)$ is also a solution.

3.1. Solutions of (18) Using the Bernoulli Equation as the Simplest Equation. The balancing procedure yields $n=1$. Thus, the solution of (18) is of the form

$$
v(\xi)=A_{0}+A_{1} H(\xi) .
$$

Substituting (19) into (18) and making use of the Bernoulli equation (5) and then equating the coefficients of the functions $H^{i}(\xi)$ to zero, we obtain an algebraic system of equations in terms of $A_{i}(i=0,1), a$, and $b$. Solving this system of algebraic equations, with the aid of Maple, one possible set of values of $A_{i}(i=0,1), a$, and $b$ is

$$
A_{0}= \pm i, \quad A_{1}= \pm \frac{2 b i}{a}, \quad \delta= \pm \sqrt{\frac{1-c^{2}}{c^{2}-\alpha^{2}}}
$$

Therefore, using solutions (6) of (5), ansatz (19), we obtain the following exact solution of (18):

$$
\begin{aligned}
& v_{1}(\xi)= \pm i \mp \frac{2 i D_{1}}{D_{1}+\cosh (a(\xi+C))-\sinh (a(\xi+C))}, \\
& v_{2}(\xi)= \pm i \mp \frac{2 i(\cosh (a(\xi+C))+\sinh (a(\xi+C)))}{D_{2}+\cosh (a(\xi+C))+\sinh (a(\xi+C))} .
\end{aligned}
$$

Substituting (21) into (17) with (12), the exact traveling wave solution to (1) can be written as

$$
\begin{aligned}
& u_{1}(\xi) \\
& =\mp\left(i\left(\left(D_{1}^{2}-1\right) \cosh (a(\xi+C))+\left(D_{1}^{2}+1\right) \sinh (a(\xi+C))\right)\right) \\
& \quad \times\left(2 a^{2}\left(c^{2}-\alpha^{2}\right) \mu^{2} D_{1}\right)^{-1}+C_{1} \xi+C_{2}, \\
& \omega_{1}(\xi) \\
& =\mp\left(i \left(\left(D_{1}^{2}-1\right) \cosh (a(\xi+C))\right.\right. \\
& \left.\left.\quad+\left(D_{1}^{2}+1\right) \sinh (a(\xi+C))\right)\right) \\
& \quad \times\left(2 a^{2}\left(c^{2}-\alpha^{2}\right) \mu^{2} D_{1}\right)^{-1}+C_{1} \xi+C_{2}-\varphi_{1},
\end{aligned}
$$

$\varphi_{1}$

$$
=2 \arctan \left( \pm i \mp \frac{2 i D_{1}}{D_{1}+\cosh (a(\xi+C))-\sinh (a(\xi+C))}\right),
$$

where $\xi=\mu(x-c t), \delta= \pm \sqrt{\left(1-c^{2}\right) /\left(c^{2}-\alpha^{2}\right)}$, and $D_{1}, a, C$, $C_{1}, C_{2}$ are arbitrary parameters.
Now, to obtain some special cases of the above solutions, we set $D_{1}=i, a=1, C=0$, “ \pm ” take “+”; we have

$$
\begin{gathered}
u_{2}(\xi)=\frac{\delta^{2} \cosh (\xi)}{\mu^{2}\left(c^{2}-1\right)}+C_{1} \xi+C_{2}, \\
\omega_{2}(\xi)=\frac{\delta^{2} \cosh (\xi)}{\mu^{2}\left(c^{2}-1\right)}+C_{1} \xi+C_{2}-\varphi_{2}, \\
\varphi_{2}=2 \arctan \left(-i-\frac{2}{i+\cosh (\xi)-\sinh (\xi)}\right) \\
=2 \arctan \left(\frac{-1+i \sinh (\xi)}{\cosh (\xi)}\right),
\end{gathered}
$$

where $\xi=\mu(x-c t), c= \pm \sqrt{\left(1+\delta^{2} \alpha^{2}\right) /\left(1+\delta^{2}\right)}$.

The equations in (24) are the same as those in (36) of [7]. If we set $D_{1}=1, a=1, C=0$, we have

$$
\begin{gathered}
u_{3}(\xi)=\mp \frac{i \delta^{2} \sinh (\xi)}{\mu^{2}\left(c^{2}-1\right)}+C_{1} \xi+C_{2}, \\
\omega_{3}(\xi)=\mp \frac{i \delta^{2} \sinh (\xi)}{\mu^{2}\left(c^{2}-1\right)}+C_{1} \xi+C_{2}-\varphi_{3}, \\
\varphi_{3}=\mp 2 \arctan \left(i-\frac{2 i}{1+\cosh (\xi)-\sinh (\xi)}\right) \\
= \pm 2 i \operatorname{arctanh}\left(\frac{\sinh (\xi)}{1+\cosh (\xi)}\right),
\end{gathered}
$$

where $\xi=\mu(x-c t), c= \pm \sqrt{\left(1+\delta^{2} \alpha^{2}\right) /\left(1+\delta^{2}\right)}$.

The equations in (25) are the same as those in (32) of [7].

Substituting (22) into (17) with (12), the exact traveling wave solution to (1) can be written as

$$
\begin{aligned}
& u_{4}(\xi) \\
& = \pm\left(i \left(\left(D_{2}^{2}-1\right) \cosh (a(\xi+C))\right.\right. \\
& \left.\left.-\left(D_{2}^{2}+1\right) \sinh (a(\xi+C))\right)\right) \\
& \times\left(2 a^{2}\left(c^{2}-\alpha^{2}\right) \mu^{2} D_{2}\right)^{-1}+C_{1} \xi+C_{2}, \\
& \omega_{4}(\xi) \\
& = \pm\left(i \left(\left(D_{2}^{2}-1\right) \cosh (a(\xi+C))\right.\right. \\
& \left.\left.-\left(D_{2}^{2}+1\right) \sinh (a(\xi+C))\right)\right) \\
& \times\left(2 a^{2}\left(c^{2}-\alpha^{2}\right) \mu^{2} D_{2}\right)^{-1}+C_{1} \xi+C_{2}-\varphi_{4},
\end{aligned}
$$

$\varphi_{4}$

$$
=2 \arctan \left( \pm i \mp \frac{2 i(\cosh (a(\xi+C))+\sinh (a(\xi+C)))}{D_{2}+\cosh (a(\xi+C))+\sinh (a(\xi+C))}\right),
$$

where $\xi=\mu(x-c t), \delta= \pm \sqrt{\left(1-c^{2}\right) /\left(c^{2}-\alpha^{2}\right)}$. 
Substituting (19) along with (9) into (18) and setting all the coefficients of powers $H^{i}(\xi)$ to be zero, then we obtain a system of nonlinear algebraic equations and by solving it, we obtain

$$
A_{0}= \pm i, \quad A_{1}=\mp \frac{2 i}{A}, \quad \delta= \pm \sqrt{\frac{1-c^{2}}{c^{2}-\alpha^{2}}}
$$

Therefore, using solutions (10) and (11) of (9), ansatz (19), we obtain the following exact solution of (18):

$$
v_{3}(\xi)=\mp i \tanh \left(\frac{A}{2}(\xi+C)\right) .
$$

Then the exact solution to (1) can be written as

$$
\begin{gathered}
u_{5}(\xi)=\mp \frac{i \sinh (A(\xi+C))}{A^{2} \mu^{2}\left(c^{2}-\alpha^{2}\right)}+C_{1} \xi+C_{2}, \\
\omega_{5}(\xi)=\mp \frac{i \sinh (A(\xi+C))}{A^{2} \mu^{2}\left(c^{2}-\alpha^{2}\right)}+C_{1} \xi+C_{2}-\varphi_{5}, \\
\varphi_{5}=2 \arctan \left(\mp i \tanh \left(\frac{A}{2}(\xi+C)\right)\right),
\end{gathered}
$$

where $\xi=\mu(x-c t), \delta= \pm \sqrt{\left(1-c^{2}\right) /\left(c^{2}-\alpha^{2}\right)}$, and $A, C, C_{1}$, $C_{2}$ are arbitrary parameters.

Now, to obtain some special cases of the above solutions, we set $A=2, C=0$; we have

$$
\begin{gathered}
u_{6}(\xi)=\mp \frac{i \delta^{2} \sinh (2 \xi)}{4 \mu^{2}\left(1-c^{2}\right)}+C_{1} \xi+C_{2}, \\
\omega_{6}(\xi)=\mp \frac{i \delta^{2} \sinh (2 \xi)}{4 \mu^{2}\left(1-c^{2}\right)}+C_{1} \xi+C_{2}-\varphi_{6}, \\
\varphi_{6}=2 \arctan (\mp i \tanh (\xi))= \pm 2 i \operatorname{arctanh}(\tanh (\xi)),
\end{gathered}
$$

where $\xi=\mu(x-c t), c= \pm \sqrt{\left(1+\delta^{2} \alpha^{2}\right) /\left(1+\delta^{2}\right)}$.

The equations in (30) are the same as those in (31) of [7].

3.2. Solutions of (18) Using Riccati Equation as the Simplest Equation. The balancing procedure yields $n=1$. Thus, the solution of (18) is of the form

$$
v(\xi)=B_{0}+B_{1} H(\xi)
$$

Substituting (31) into (18) and making use of the Riccati Equation (7) and then equating the coefficients of the functions $H^{i}(\xi)$ to zero, we obtain an algebraic system of equations in terms of $B_{i}(i=0,1), a, b$, and $s$. Solving this system of algebraic equations, with the aid of Maple, one possible set of values of $B_{i}(i=0,1), a, b$, and $s$ is

$$
B_{1}=\frac{2 a B_{0}}{b}, \quad s=\frac{b^{2}\left(1+B_{0}^{2}\right)}{4 a B_{0}^{2}}, \quad \delta= \pm \sqrt{\frac{1-c^{2}}{c^{2}-\alpha^{2}}} \text {. }
$$

Therefore, using solutions (8) of (7), ansatz (31), we obtain the following exact solution of (18):

$$
\begin{gathered}
v_{1}(\xi)=\tan \left(\frac{b(\xi+C)}{2 B_{0}}\right), \\
v_{2}(\xi)=-\frac{C b \sin \left(\left(b / 2 B_{0}\right) \xi\right)+2 B_{0} a \cos \left(\left(b / 2 B_{0}\right) \xi\right)}{-C b \cos \left(\left(b / 2 B_{0}\right) \xi\right)+2 B_{0} a \sin \left(\left(b / 2 B_{0}\right) \xi\right)} .
\end{gathered}
$$

Substituting (33) into (17) with (12), the exact traveling wave solution to (1) can be written as

$$
\begin{gathered}
u_{1}(\xi)=-\frac{B_{0}^{2} \sin \left(\left(b / B_{0}\right)(\xi+C)\right)}{\left(c^{2}-\alpha^{2}\right) \mu^{2} b^{2}}+C_{1} \xi+C_{2}, \\
\omega_{1}(\xi)=-\frac{B_{0}^{2} \sin \left(\left(b / B_{0}\right)(\xi+C)\right)}{\left(c^{2}-\alpha^{2}\right) \mu^{2} b^{2}}+C_{1} \xi+C_{2}-\varphi_{1}, \\
\varphi_{1}=2 \arctan \left(\tan \left(\frac{b}{2 B_{0}}(\xi+C)\right)\right)
\end{gathered}
$$

where $\xi=\mu(x-c t), \delta= \pm \sqrt{\left(1-c^{2}\right) /\left(c^{2}-\alpha^{2}\right)}$.

Substituting (34) into (17) with (12), the exact traveling wave solution to (1) can be written as

$$
\begin{aligned}
& u_{2}(\xi) \\
& =-\left(C^{2} B_{0}^{2} \sin \left(\frac{b}{B_{0}} \xi\right)\right. \\
& \left.\quad+\frac{4 a B_{0}^{3}}{b^{2}}\left(b C \cos \left(\frac{b}{B_{0}} \xi\right)-B_{0} a \sin \left(\frac{b}{B_{0}} \xi\right)\right)\right) \\
& \quad \times\left(\left(c^{2}-\alpha^{2}\right) \mu^{2}\left(C^{2} b^{2}+4 B_{0}^{2} a^{2}\right)\right)^{-1}+C_{1} \xi+C_{2},
\end{aligned}
$$

$\omega_{2}(\xi)$

$$
\begin{gathered}
=-\left(C^{2} B_{0}^{2} \sin \left(\frac{b}{B_{0}} \xi\right)\right. \\
\left.\quad+\frac{4 a B_{0}^{3}}{b^{2}}\left(b C \cos \left(\frac{b}{B_{0}} \xi\right)-B_{0} a \sin \left(\frac{b}{B_{0}} \xi\right)\right)\right) \\
\quad \times\left(\left(c^{2}-\alpha^{2}\right) \mu^{2}\left(C^{2} b^{2}+4 B_{0}^{2} a^{2}\right)\right)^{-1}+C_{1} \xi+C_{2}-\varphi_{2}, \\
\varphi_{2}=2 \arctan \left(-\frac{C b \sin \left(\left(b / 2 B_{0}\right) \xi\right)+2 B_{0} a \cos \left(\left(b / 2 B_{0}\right) \xi\right)}{-C b \cos \left(\left(b / 2 B_{0}\right) \xi\right)+2 B_{0} a \sin \left(\left(b / 2 B_{0}\right) \xi\right)}\right),
\end{gathered}
$$

where $\xi=\mu(x-c t), \delta= \pm \sqrt{\left(1-c^{2}\right) /\left(c^{2}-\alpha^{2}\right)}$.

Remark 2. Compared with [7], the exact solutions of this paper are more general, such that when $D_{1}=i, a=1$, and $C=0$ in (23), the solutions become as those in (36) of [7]. When $D_{1}=1, a=1$, and $C=0$ in (23), the solutions become as those in (32) of [7]. When $A=2$ and $C=0$ of (29), the solutions become as those in (31) of [7]. There are many such examples; thus, it is easy to see that the study of [7] is a special 
case in this paper. So the exact solutions of this paper are more general, and all the solutions are new solutions which are not reported in the relevant literature reported.

\section{Conclusions}

In this paper, we obtained some exact solutions of the coupled sine-Gordon equations by using the simplest equation method. The Bernoulli equation and Riccati equation have been used as the simplest equation. The solutions obtained may be significant and important for the explanation of some practical physical problems. The method may also be applied to other nonlinear partial differential equations. Also, we have verified that the solutions that we have found are indeed solutions to the original nonlinear evolution equations.

\section{Conflict of Interests}

The author declares that there is no conflict of interests regarding the publication of this paper.

\section{Acknowledgment}

This work was supported by the National Natural Science Foundation of China (11161020 and 11361023), the Natural Science Foundation of Yunnan Province (2011FZ193 and 2013FZ117), and the Natural Science Foundation of Education Committee of Yunnan Province (2012Y452 and 2013C079).

\section{References}

[1] K. R. Khusnutdinova and D. E. Pelinovsky, "On the exchange of energy in coupled Klein-Gordon equations," Wave Motion, vol. 38, no. 1, pp. 1-10, 2003.

[2] T. A. Kontorova and Y. I. Frenkel, "On the theory of plastic deformation and twinning I, II," Zhurnal Eksperimental'NoI I TeoreticheskoI Fiziki, vol. 8, pp. 89-95, 1938.

[3] O. M. Braun and Y. S. Kivshar, "Nonlinear dynamics of the Frenkel-Kontorova model," Physics Reports, vol. 306, no. 1-2, p. $108,1998$.

[4] S. Yomosa, "Soliton excitations in deoxyribonucleic acid (DNA) double helices," Physical Review A, vol. 27, no. 4, pp. 2120-2125, 1983.

[5] A. H. Salas, "Exact solutions of coupled sine-Gordon equations," Nonlinear Analysis: Real World Applications, vol. 11, no. 5, pp. 3930-3935, 2010.

[6] Y.-M. Zhao, H. Liu, and Y.-J. Yang, "Applications of the Jacobi elliptic function expansion method for obtaining travelling wave solutions of coupled sine-Gordon equations," Mathematical Sciences Research Journal, vol. 15, no. 3, pp. 80-90, 2011.

[7] Y.-M. Zhao, H. Liu, and Y.-J. Yang, "Exact solutions for the coupled Sine-Gordon equations by a new hyperbolic auxiliary function method," Applied Mathematical Sciences, vol. 5, no. 3336, pp. 1621-1629, 2011.

[8] Y.-M. Zhao, W. Li, and Y.-J. Yang, "New exact solutions of coupled sine-Gordon equations using symbolic computation," Mathematical Sciences Research Journal, vol. 14, no. 4, pp. 7986, 2010.

[9] M. Wang, "Exact solutions for a compound KdV-Burgers equation," Physics Letters A, vol. 213, no. 5-6, pp. 279-287, 1996.
[10] M. L. Wang, Y. B. Zhou, and Z. B. Li, "Application of a homogeneous balance method to exact solutions of nonlinear equations in mathematical physics," Physics Letters A, vol. 216, no. 1-5, pp. 67-75, 1996.

[11] Sirendaoreji and S. Jiong, "Auxiliary equation method for solving nonlinear partial differential equations," Physics Letters A, vol. 309, no. 5-6, pp. 387-396, 2003.

[12] S. Zhang and T. Xia, "A generalized new auxiliary equation method and its applications to nonlinear partial differential equations," Physics Letters A, vol. 363, no. 5-6, pp. 356-360, 2007.

[13] X.-H. Wu and J.-H. He, "Solitary solutions, periodic solutions and compacton-like solutions using the Exp-function method," Computers \& Mathematics with Applications. An International Journal, vol. 54, no. 7-8, pp. 966-986, 2007.

[14] X.-H. Wu and J.-H. He, "EXP-function method and its application to nonlinear equations," Chaos, Solitons \& Fractals, vol. 38, no. 3, pp. 903-910, 2008.

[15] S. B. Leble and N. V. Ustinov, "Darboux transforms, deep reductions and solitons," Journal of Physics A, vol. 26, no. 19, pp. 5007-5016, 1993

[16] H.-C. Hu, X.-Y. Tang, S.-Y. Lou, and Q.-P. Liu, "Variable separation solutions obtained from Darboux transformations for the asymmetric Nizhnik-Novikov-Veselov system," Chaos, Solitons \& Fractals, vol. 22, no. 2, pp. 327-334, 2004.

[17] H. A. Abdusalam, "On an improved complex tanh-function method," International Journal of Nonlinear Sciences and Numerical Simulation, vol. 6, no. 2, pp. 99-106, 2005.

[18] M. L. Wang, X. Z. Li, and J. L. Zhang, "The $G^{\prime} / G$-expansion method and travelling wave solutions of nonlinear evolution equations in mathematical physics," Physics Letters A, vol. 372, no. 4, pp. 417-423, 2008.

[19] S. M. Guo and Y. B. Zhou, "The extended $G^{\prime} / G$-expansion method and its applications to the Whitham-Broer-Kauplike equations and coupled Hirota-Satsuma KdV equations," Applied Mathematics and Computation, vol. 215, no. 9, pp. 32143221, 2010.

[20] N. A. Kudryashov, "Exact solitary waves of the Fisher equation," Physics Letters A, vol. 342, no. 1-2, pp. 99-106, 2005.

[21] N. A. Kudryashov, "Simplest equation method to look for exact solutions of nonlinear differential equations," Chaos, Solitons and Fractals, vol. 24, no. 5, pp. 1217-1231, 2005.

[22] N. K. Vitanov and Z. I. Dimitrova, "Application of the method of simplest equation for obtaining exact traveling-wave solutions for two classes of model PDEs from ecology and population dynamics," Communications in Nonlinear Science and Numerical Simulation, vol. 15, no. 10, pp. 2836-2845, 2010.

[23] N. K. Vitanov, Z. I. Dimitrova, and H. Kantz, "Modified method of simplest equation and its application to nonlinear PDEs," Applied Mathematics and Computation, vol. 216, no. 9, pp. 25872595, 2010.

[24] C. M. Khalique, "Exact explicit solutions and conservation laws for a coupled Zakharov-Kuznetsov system," Mathematical Problems in Engineering, vol. 2013, Article ID 461327, 5 pages, 2013. 


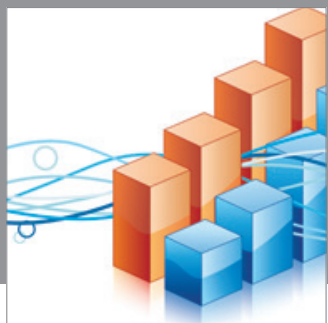

Advances in

Operations Research

mansans

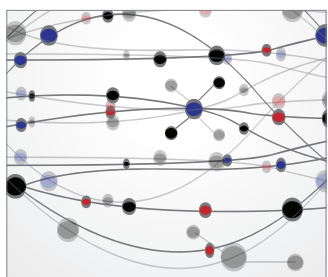

The Scientific World Journal
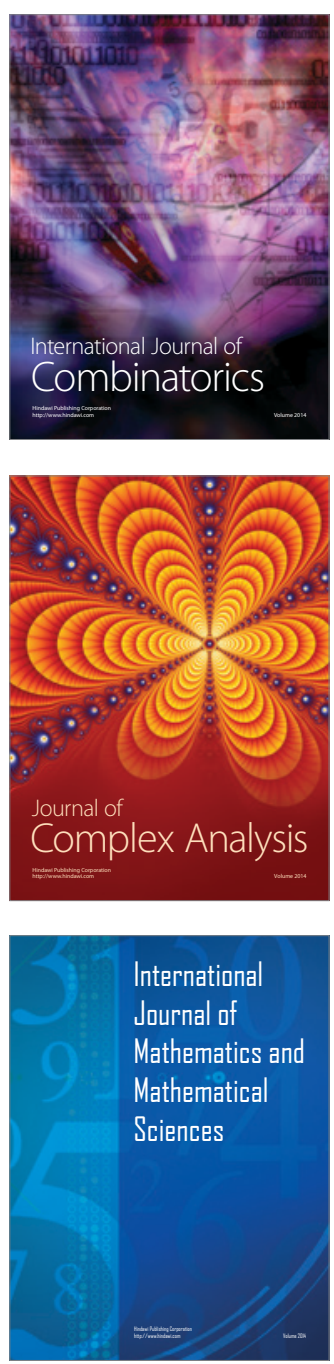
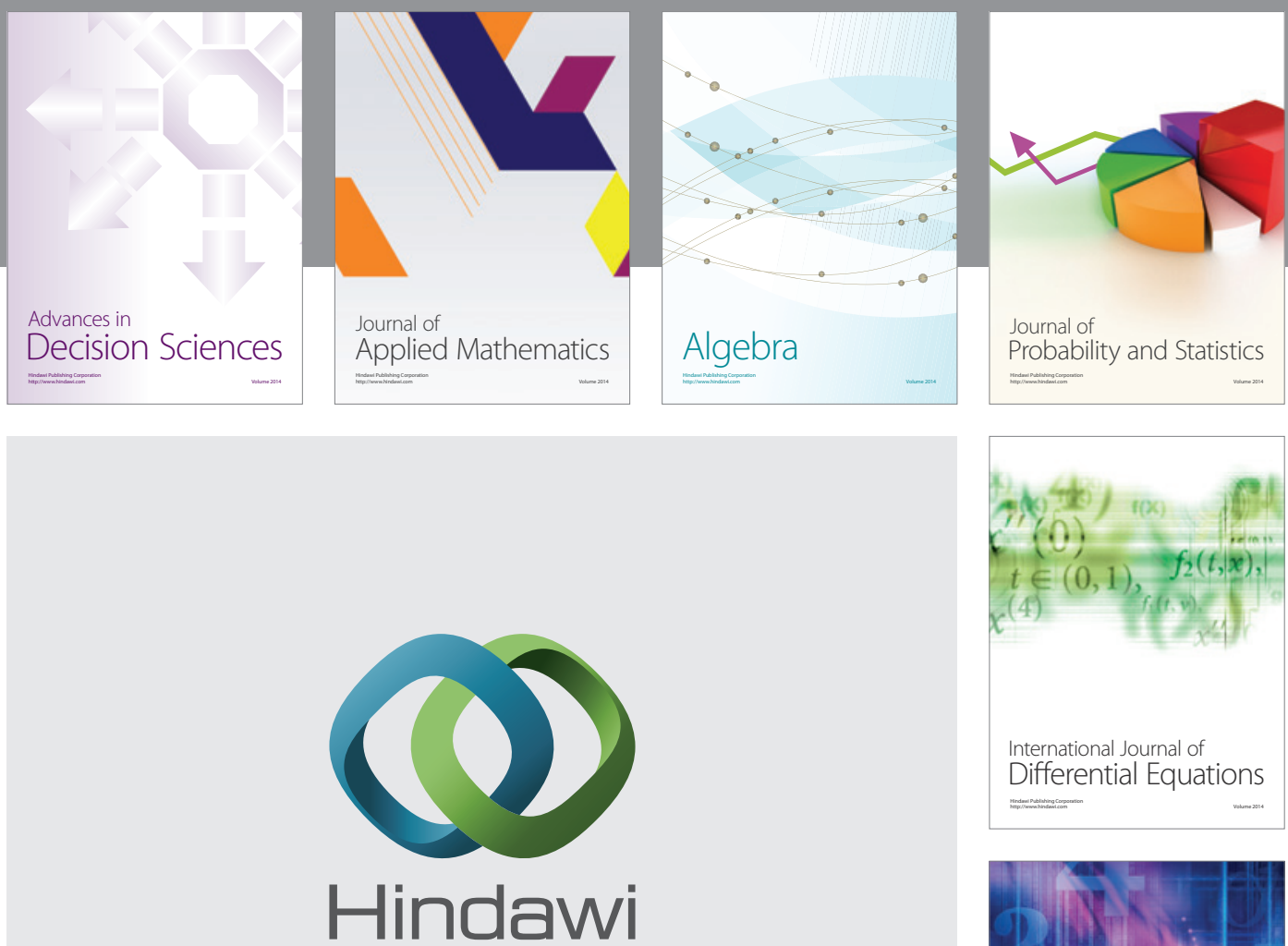

Submit your manuscripts at http://www.hindawi.com
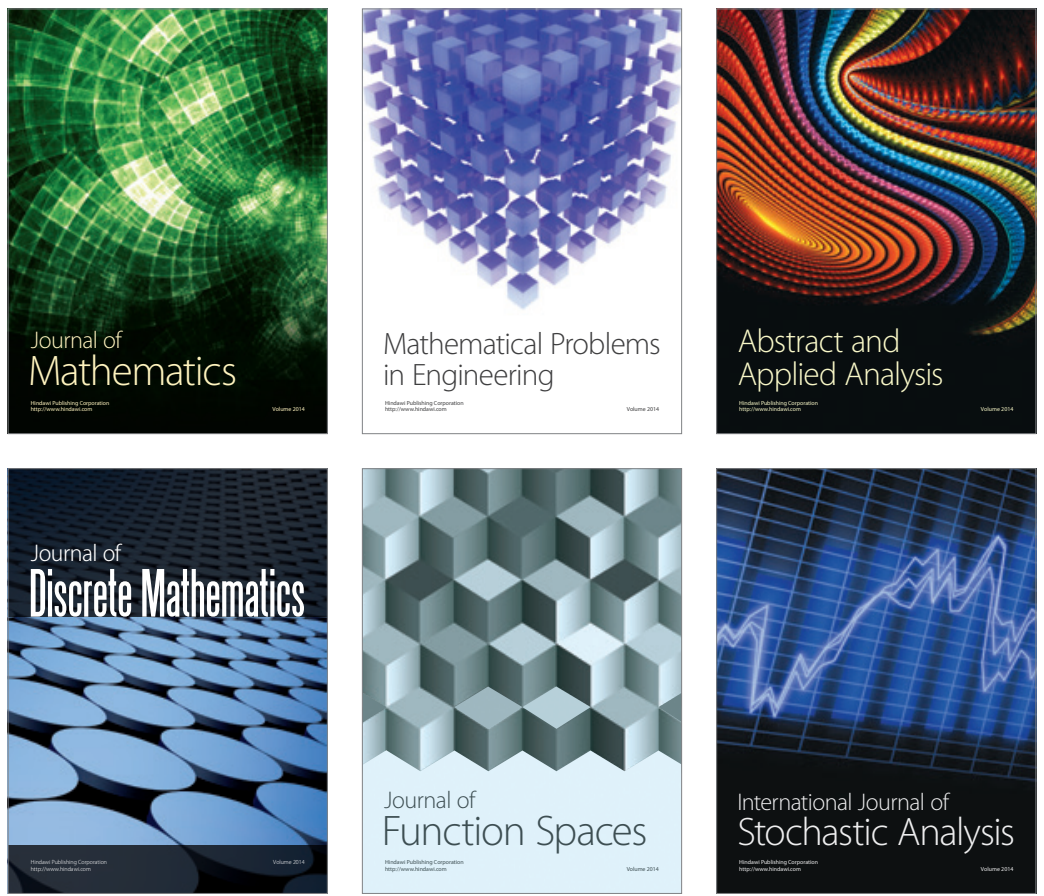

Journal of

Function Spaces

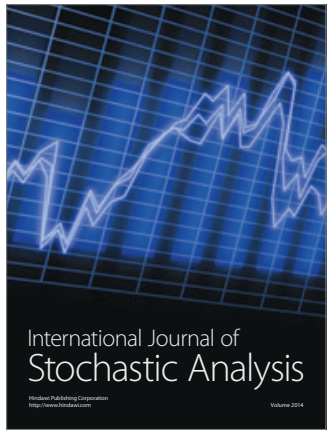

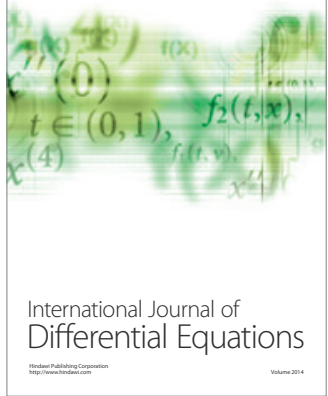
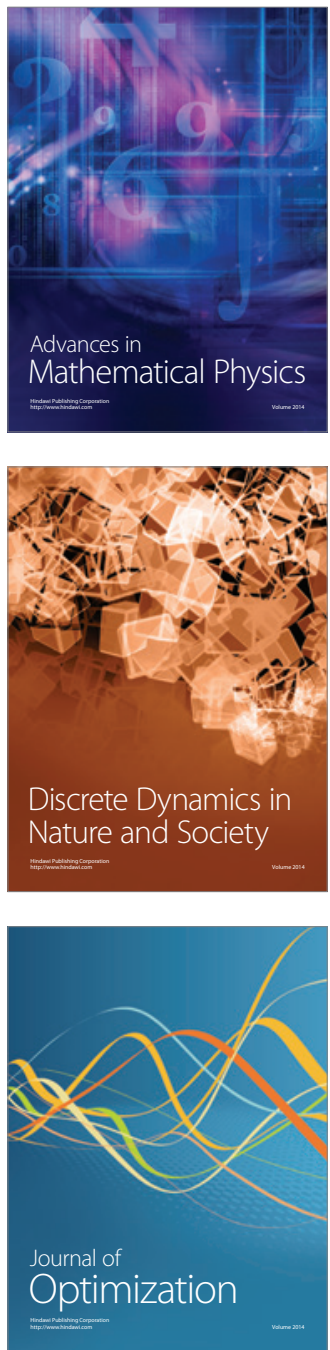